\title{
Many-body properties of a disordered charged Bose gas superlattice
}

\author{
B. TANATAR \\ Department of Physics, Bilkent University, Bilkent, 06533 Ankara, Turkey \\ A. K. DAS \\ Department of Physics, Dalhousie University, Halifax, Nova Scotia, Canada B3H 3J5
}

(Received 9 December 1999)

\begin{abstract}
We study some many-body properties of a disordered charged Bose gas (CBG) superlattice-an infinite array of CBG layers each of which containing disorder. The latter is assumed to cause collisions with the charged bosons, the effect of collisions being taken into account through a number-conserving relaxation time approximation incorporated within the random phase approximation (RPA) at $T=0$. We go beyond the RPA and include a local-field correction $G\left(q, q_{z}\right)$ which is assumed to be collision independent, as an approximation. The resulting density-density correlation function is then used to calculate a number of many-body quantities of physical interest, e.g. (a) collective modes, (b) static structure factor, (c) energy-loss function, (d) plasmon density of states, and (e) groundstate energy. The effects of collisions on these quantities are discussed, and the results are compared with the corresponding results for an electron gas superlattice.
\end{abstract}

Key words: many-body properties, charged Bose gas superlattices.

(c) 2000 Academic Press

Studies on low-dimensional charged Bose systems have been gaining attention because of their possible role in the theoretical understanding of a class of high-temperature superconductors [1-4]. There has been a great deal of interest in two-dimensional electronic systems such as electrons at the interfaces of semiconductors and similar devices owing to their potential applications and from a fundamental point of view [5]. Charged bosons are similar to electrons in their interactions among themselves but differ in the statistics they obey. The possibility of realizing a charged boson system in semiconductor heterostructures was also pointed out [6]. Many-body properties of a charged Bose gas [7] (CBG) are also interesting in their own right, as they help our understanding of correlation effects in relation to electron gas models. Recently, we have discussed the collective modes (plasmons) and screened interactions in a CBG system in the absence and the presence of disorder within a phenomenological approach [8]. Most recent work $[9,10]$ concentrated on the correlation effects in various charged boson systems using techniques going beyond the simple random-phase approximation (RPA).

It is known $[8,11]$ that disorder effects, taken into account in the form of carrier-impurity collisions, severely constrain plasmon propagation in a single-layer electron and charged Bose gases, and that this constraint may be partially remedied in double-layer systems. The main finding is that in lower dimensions (lower than three) the plasmon dispersion in the absence of disorder becomes gapless and develops a cut-off in wave vector in the presence of disorder; collective excitations having wave vectors less than the cut-off value cannot propagate. 
If a CBG model can indeed be applicable to a class of high- $T_{c}$ superconductors then disorder in the CBG superlattice is of both theoretical and practical interest. In this paper we present a many-body calculation in which we attempt to include some effects of disorder in a CBG superlattice, in a model that goes beyond the previously studied RPA. One of our objectives is to study the effect of local-field corrections on the manybody properties. We assume that at $T=0$, all the particles are in a condensate state both in the absence and in the presence of disorder. We neglect the depletion of the condensate due to interactions and disorder. It was argued that disorder cannot completely deplete the condensate [12]. For the clean (disorder-free) and disordered two-dimensional CBG systems, we have reported [8] that the plasmon characteristics are similar to those found for the corresponding electron gas systems. Screening properties of a disordered Bose condensate were also investigated within the memory-function formalism [13]. Our approach is phenomenological in the sense that disorder is treated within the number-conserving relaxation-time approximation, described by a parameter $\tau$ appearing in the density-density response function. It can be shown that a constant memory function gives the same result as the number-conserving relaxation-time approximation. We do not specify the origin of disorder, but it may arise from scattering mechanisms such as impurity scattering. We also note that our calculations are limited to the regime of weak disorder. As the strength of disorder increases the charged Bose system is expected to undergo a superfluid-insulator transition $[2,13]$ in which case our model does not apply.

Layered electron systems in a model consisting of a periodic array of two-dimensional electron gases have been extensively studied $[14,15]$. Boson superlattices and single-layer systems, especially in the context of high- $T_{c}$ superconductivity, have also been investigated with and without the disorder [16]. We consider a disordered superlattice charge Bose gas. The density-density response function for an interacting system of bosons is given by $[16,17] \chi(q, \omega ; \tau)=\chi_{0}(q, \omega ; \tau) /\left[1-V\left(q, q_{z}\right)\left[1-G\left(q, q_{z}\right)\right] \chi_{0}(q, \omega ; \tau)\right]$, in which the response function for a non-interacting system with disorder in the number-conserving scheme [18] is shown to be

$$
\chi_{0}(q, \omega ; \tau)=\frac{\omega_{+} \chi_{0}\left(q, \omega_{+}\right)}{\omega+(i / \tau) \chi_{0}\left(q, \omega_{+}\right) / \chi_{0}(q, 0)}=\frac{2 n \varepsilon_{q}}{\omega \omega_{+}-\varepsilon_{q}^{2}},
$$

(we put $\hbar=1$ ) where $\omega_{+}=\omega+i / \tau$ ( $\tau$ is the relaxation time which will be treated as a phenomenological parameter), $\varepsilon_{q}=q^{2} / 2 m$ is the free-particle energy, and $\chi_{0}(q, 0)$ is the static susceptibility. In the limit $\tau \rightarrow \infty, \chi_{0}(q, \omega ; 1 / \tau \rightarrow 0)$ becomes the collisionless $\chi_{0}(q, \omega)$. The Coulomb interaction potential for charged particles in a superlattice is given by [14]

$$
V\left(q, q_{z}\right)=\frac{2 \pi e^{2}}{q} \frac{\sinh q d}{\cosh q d-\cos q_{z} d},
$$

where $q$ and $q_{z}\left(-\pi / d \leq q_{z} \leq \pi / d\right)$ are in-plane and perpendicular wave vectors, respectively, and $d$ is the distance between layers. As stated earlier, we have included the local-field correction as described by $G\left(q, q_{z}\right)$ to account for the correlation effects. Setting $G\left(q, q_{z}\right)=0$, we recover the usual RPA. For layered electron gas and charged Bose systems, $G\left(q, q_{z}\right)$ has been calculated in a variety of approximations $[15,16]$. In this paper, we use the Hubbard approximation, in which the local-field factor takes the form [16]

$$
G_{H}\left(q, q_{z}\right)=r_{s}^{2 / 3} \frac{q}{\sqrt{q^{2}+q_{s}^{2}}} \frac{\left(\cosh q d-\cos q_{z} d\right)}{\sinh q d} F_{H}\left(q, q_{z}\right),
$$

with

$$
F_{H}\left(q, q_{z}\right)=1-\frac{\left(q_{z} / d\right) \sin q_{z} d \tan ^{-1}\left[\sinh \left(d \sqrt{q^{2}+q_{z}^{2}}\right)\right]}{q^{2}+q_{z}^{2}} .
$$

Strictly, $G_{H}\left(q, q_{z}\right)$ is only valid for $r_{s} \leq 1$. In the low-density regime $\left(r_{s} \gg 1\right)$, a more sophisticated calculation [15, 16] of $G\left(q, q_{z}\right)$ is necessary. $G_{H}\left(q, q_{z}\right)$ as given by eqn (3) does not include effects of disorder. Our objective in this paper is to see how this level of approximation beyond RPA affects some many-body properties of a CBG superlattice. We employ the natural inverse length scale given by the screening wave 


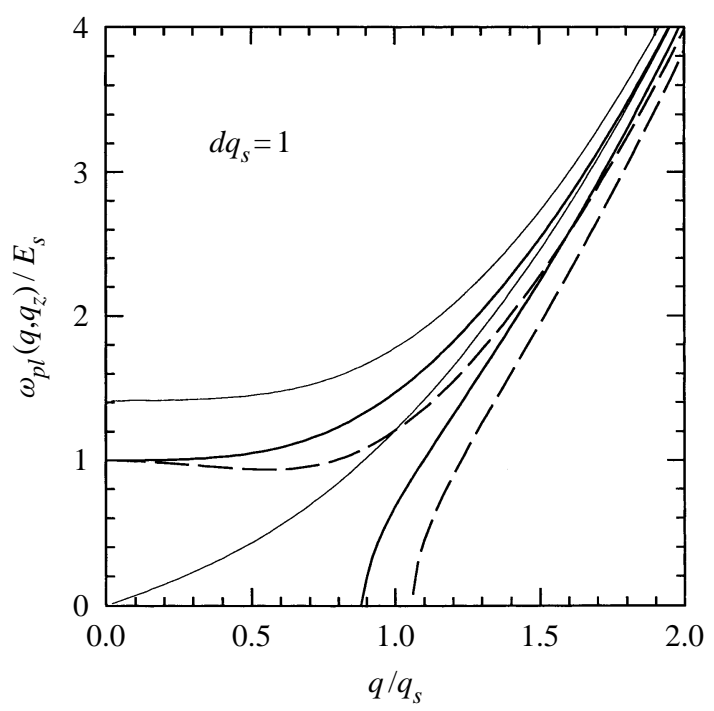

Fig. 1. Plasmon dispersions of a disordered CBG superlattice in the RPA for $1 /\left(2 \tau E_{S}\right)^{2}=0$ (thin solid curves) and $1 /\left(2 \tau E_{S}\right)^{2}=1$ (thick solid curves). Upper and lower curves indicate $q_{z} d=0$ and $\pi$, respectively. The combined effect of the local field corrections $G_{H}\left(q, q_{z}\right)$ and disorder $\left(1 /\left(2 \tau E_{S}\right)^{2}=1\right)$ on the collective modes is indicated by the dashed curves. In all curves we take $d q_{s}=1$.

vector defined as $q_{s}=\left(8 \pi n / a_{B}\right)^{1 / 3}$ where $a_{B}$ is the Bohr radius. The dimensionless density parameter $r_{s}^{2}=1 /\left(\pi n a_{B}^{2}\right)$, is then related to the screening wave vector through $q_{s} a_{B}=2 / r_{s}^{2 / 3}$. Energies are expressed in units of $E_{s}=q_{s}^{2} / 2 m$.

The first quantity that we consider is the plasmon dispersion relation obtained from the pole of the densitydensity response function to read

$$
\omega_{\mathrm{pl}}\left(q, q_{z} ; \tau\right)=\left[\varepsilon_{q}^{2}+2 n \varepsilon_{q} V\left(q, q_{z}\right)\left[1-G\left(q, q_{z}\right)\right]-\frac{1}{4 \tau^{2}}\right]^{1 / 2}-\frac{i}{2 \tau} .
$$

We note that the above expression is valid within and beyond the RPA for all wave vectors, since no lowfrequency or wave vector approximations were made to the density response function. Disorder generally reduces the plasmon energy which was also discussed by Gold [13]. Similar results for the single-layer electron gas have been obtained [11] for $q \ll k_{F}$, where $k_{F}$ is the Fermi wave vector. The imaginary part to the plasmon dispersion is independent of $q$ and $q_{z}$ within our model, a situation different from the electron gas case in which it is wave vector dependent. In Fig. 1 we show $\omega_{\mathrm{pl}}\left(q, q_{z}\right)$ within the RPA for clean (thin solid curves) and disordered (thick solid curves) cases as a function of the in-plane wave vector $q$ for $d q_{s}=1$. Upper and lower curves for both cases indicate $q_{z} d=0$ and $q_{z} d=\pi$, respectively, the boundaries of possible plasmon excitations. For $q_{z}=0$, the plasmon energy has a gap as in a bulk fermion or boson system. The combined effect of the local-field corrections $G_{H}\left(q, q_{z}\right)\left(\right.$ at $\left.r_{s}=1\right)$ and disorder is indicated by the dashed curves. Notice that in general, $G_{H}\left(q, q_{z}\right)$ lowers the plasmon dispersion. In the $q_{z} d=0$ branch a small dip appears, whereas in the $q_{z} d=\pi$ branch the cut-off wave vector increases.

As in the case of electron gas [11], there exists a critical wave vector $q_{c}$ (for a fixed $q_{z}$ ) below which the collective modes do not propagate. The critical $q_{c}$ is obtained from the solution of

$$
q_{c}^{4}+4 n m q_{c}^{2} V\left(q_{c}, q_{z}\right)\left[1-G\left(q_{c}, q_{z}\right)\right]-\left(\frac{m}{\tau}\right)^{2}=0
$$



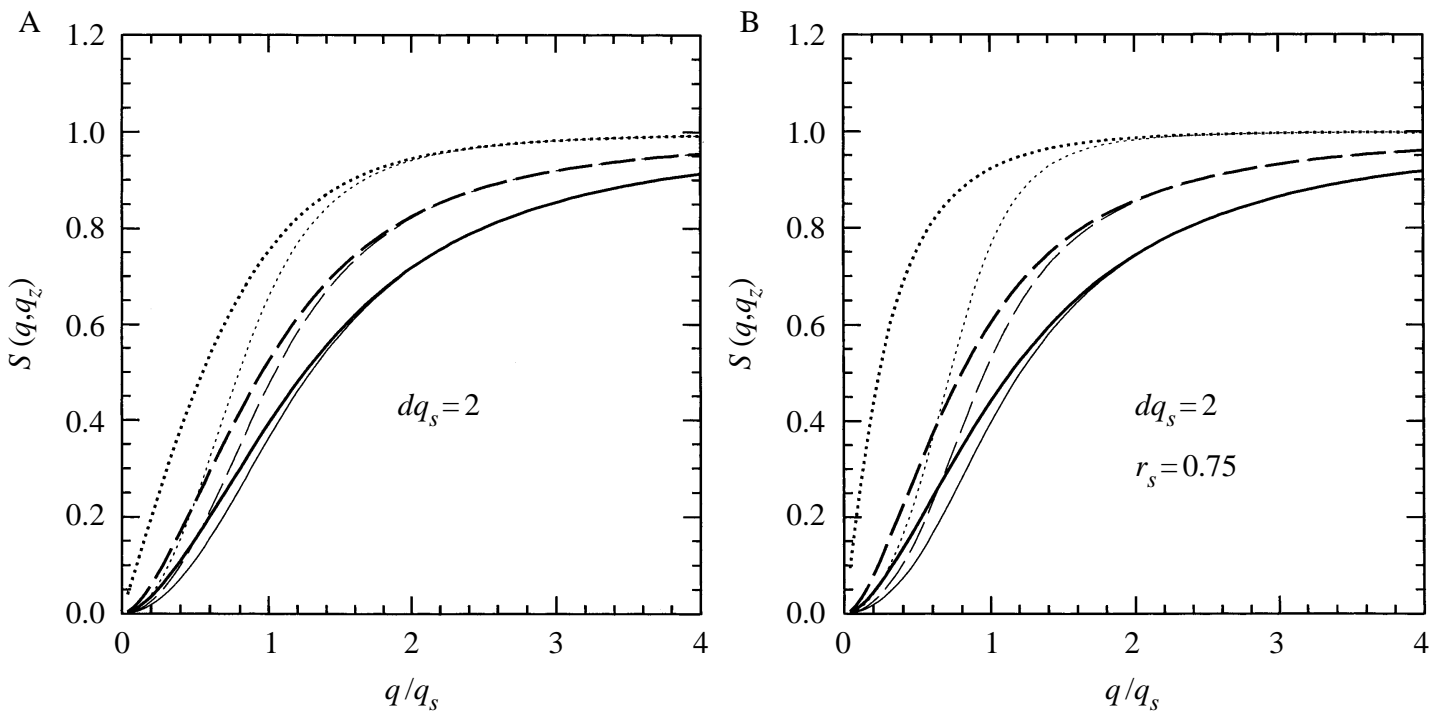

Fig. 2. Static structure factor $S\left(q, q_{z} ; \tau\right)$ for CBG superlattice within A, the RPA and B, including the local-field corrections, for $(2 \tilde{\tau})^{-2}=0,1$, and 5 shown by dotted, dashed, and solid curves, respectively. Upper (thin curves) and lower curves (thick curves) indicate $q_{z} d=\pi / 2$ and $\pi$, respectively.

for $-\pi / d \leq q_{z} \leq \pi / d$. In Fig. 1 , the lower boundary of the plasmon excitations defined by $q_{z} d=\pi$ starts off at $q_{c}$ illustrating this effect. As the degree of disorder is increased (i.e. as $1 / \tau$ gets large), it is possible to have $q_{z} d=0$, and plasmons to exhibit a gapless dispersion, a truly 2D behaviour.

The static structure factor $S\left(q, q_{z}\right)$, of a superlattice CBG is of interest because it reflects the groundstate correlations in the system. We employ the usual method of performing a frequency integral over the fluctuation-dissipation theorem [17]

$$
S\left(q, q_{z} ; \tau\right)=-\frac{1}{n \pi} \int_{0}^{\infty} d \omega \chi(q, i \omega ; \tau)
$$

where the response function has been defined above. The disorder effects enter through the parameter $\tau$, and the correlation effects through the local-field factor $G\left(q, q_{z}\right)$. The resulting expression is $S\left(q, q_{z} ; \tau\right)=$ $(1 / \pi) \varepsilon_{q} I(\Delta)$, where

$$
I(\Delta)=2 \begin{cases}\frac{1}{\sqrt{\Delta}}\left[\frac{\pi}{2}-\tan ^{-1}\left(\frac{1 / \tau}{\sqrt{\Delta}}\right)\right], & \text { for } \Delta>0, \\ \tau, & \text { for } \Delta=0, \\ \frac{1}{\sqrt{-\Delta}} \tanh ^{-1}\left(\frac{1 / \tau}{\sqrt{-\Delta}}\right), & \text { for } \Delta<0,\end{cases}
$$

and $\Delta=4\left(\varepsilon_{q}^{2}+2 n V\left(q, q_{z}\right)\left[1-G\left(q, q_{z}\right)\right] \varepsilon_{q}\right)-1 / \tau^{2}$. The above result yields the simple expression $S\left(q, q_{z}\right)=\left[1+2 n V\left(q, q_{z}\right)\left[1-G\left(q, q_{z}\right)\right] / \varepsilon_{q}\right]^{-1 / 2}$ as $\tau \rightarrow \infty$, i.e. disorder-free limit [16]. $G\left(q, q_{z}\right) \rightarrow 0$ limit gives $S\left(q, q_{z}\right)$ in the RPA. We illustrate the effect of finite $\tau$ on $S\left(q, q_{z} ; \tau\right)$ within the RPA in Fig. 2A. We find that $q_{z}=0$ and $q_{z}=\pi$ structure factors are very similar, but $q_{z}=\pi / 2$ case shows differences in the small $q$ region. As $1 / \tau$ gets large the magnitude of $S\left(q, q_{z} ; \tau\right)$ becomes smaller, and it approaches unity in the large $q$ limit in a slower fashion than that of a disorder-free $S\left(q, q_{z}\right)$. It is interesting to note that the disordered $S\left(q, q_{z} ; \tau\right)$ is a smooth function of the disorder parameter $\tau$, not reflecting the sharp cut-off $q_{c}$ in the plasmon dispersion. The dotted curves indicate the structure factor (for $q_{z}=\pi / 2$ and $q_{z}=\pi$ ) in the absence of disorder, i.e. $1 / \tau=0$, and we note the marked difference from the finite $\tau$ cases. Figure 2B 


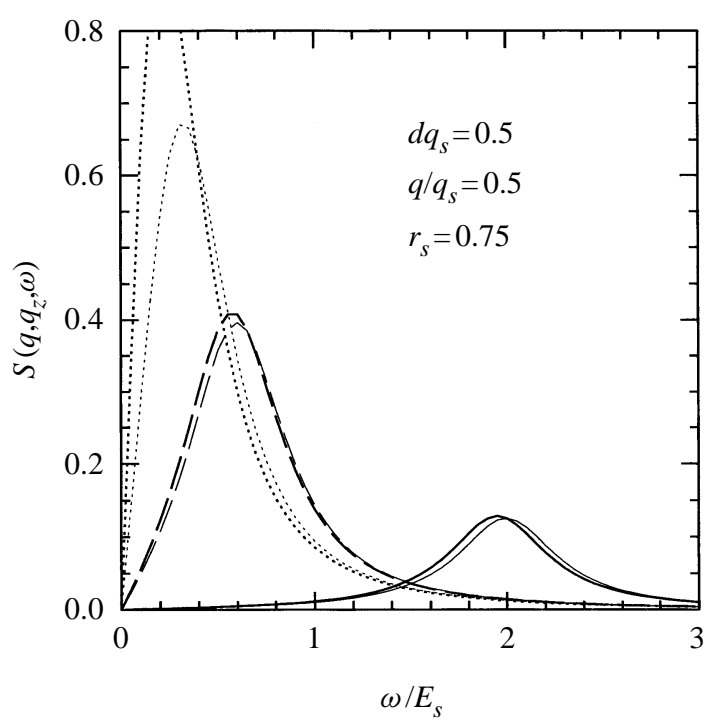

Fig. 3. The loss functions $S\left(q, q_{z} ; \omega ; \tau\right)$ as a function of $\omega$ at $q_{z}=0$ (solid curves), $q_{z} d=\pi / 2$ (dashed curves), and $q_{z} d=\pi$ (dotted curves). Thick and thin curves refer to RPA and local-field corrections, respectively.

shows the combined effect of disorder and exchange-correlation effects in the Hubbard approximation on $S\left(q, q_{z} ; \tau\right)$ at $r_{s}=0.75$. In general $G\left(q, q_{z}\right)$ affects $S\left(q, q_{z} ; \tau\right)$ in the low $q$ region. It appears that a finite $\tau$ tends to lower the magnitude of $S\left(q, q_{z} ; \tau\right)$ whereas local-field effects tend to enhance it, resulting in an approximate cancellation.

Further insight into the collective modes can be gained by considering the energy-loss function $S\left(q, q_{z}\right.$, $\omega ; \tau)$ in a layered CBG system. Using the fluctuation-dissipation theorem [17], we calculate the effects of disorder and local-field correction on the energy-loss function which is proportional to the imaginary part of the inverse response function. In Fig. 3 we show $S\left(q, q_{z}, \omega ; \tau\right)$ (in arbitrary units) as a function of $\omega$, at $q=0.5 q_{s}$ and $q_{z}=0$ (solid curves), $q_{z} d=\pi / 2$ (dashed curves), and $q_{z} d=\pi$ (dotted curves). We take the separation between the superlattice layers to be $\tilde{d} \equiv d q_{s}=0.5$ and the disorder parameter $\left(2 \tau E_{s}\right)^{-2}=0.1$. What would be delta-function peaks in the absence of disorder broaden upon the inclusion of a finite $\tau$. Thick and thin curves indicate $S\left(q, q_{z}, \omega ; \tau\right)$ calculated with and without local-field effects (at $r_{s}=0.75$ ), respectively. We find that $G_{H}\left(q, q_{z}\right)$ increases the magnitude of $S\left(q, q_{z}, \omega ; \tau\right)$, mostly affecting the $q_{z} d=\pi$ case, consistent with the static structure factor results discussed earlier.

It has been found useful to study the plasmon density of states in interpreting the photo-electron spectra in layered materials, particularly high- $T_{c}$ superconductors [19]. The plasmon density of states is defined by $[19,20]$

$$
\rho(\omega)=\sum_{q, q_{z}} \delta\left(\omega-\omega_{\mathrm{pl}}\left(q, q_{z} ; \tau\right)\right) .
$$

Using the plasmon dispersion relation in the presence of disorder and accounting for the correlations through the local-field factor, we find

$$
\begin{aligned}
\rho(\omega)= & \frac{q_{s} m}{2 \pi^{2}} y \\
& \times \int_{-\pi / \tilde{d}}^{\pi / \tilde{d}} \frac{d z x_{m}(z, y)}{4 x_{m}^{3}+\frac{\sinh x_{m} \tilde{d}+x_{m} \tilde{d} \cosh x_{m} \tilde{d}}{\cosh x_{m} \tilde{d}-\cos z \tilde{d}}[1-G]-\frac{x_{m} \tilde{d} \sinh ^{2} x_{m} \tilde{d}}{\left(\cosh x_{m} \tilde{d}-\cos x_{m} \tilde{d}\right)^{2}}[1-G]-\frac{x_{m} \sinh x_{m} \tilde{d}}{\cosh x_{m} \tilde{d}-\cos z \tilde{d}} G^{\prime}},
\end{aligned}
$$



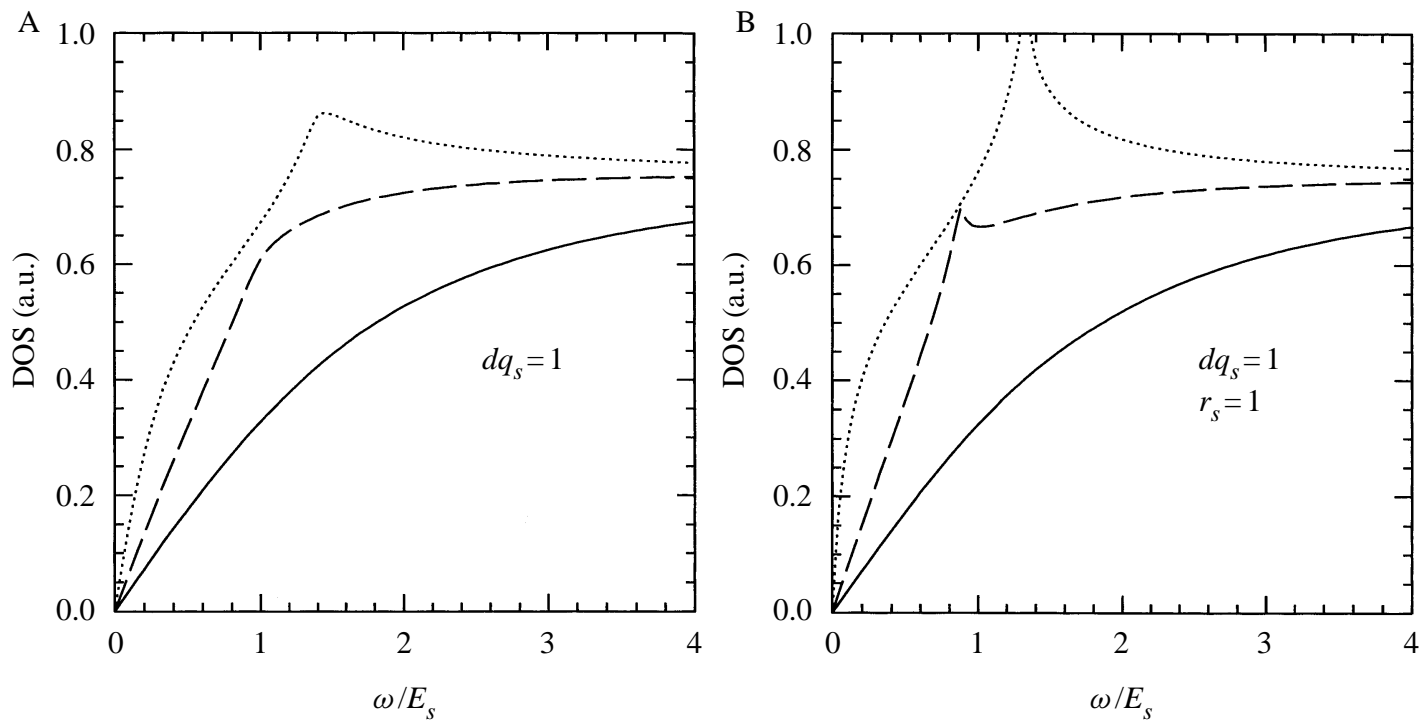

Fig. 4. Plasmon density of states in a CBG superlattice within A, the RPA and B, including the local-field corrections, for $(2 \tilde{\tau})^{-2}=0$, 1 , and 5 shown by dotted, dashed, and solid curves, respectively.

where $G^{\prime}\left(x_{m}, z\right)$ denotes the derivative of $G(x, z)$ with respect to the first argument, and $x_{m}(z, y)$ is obtained from

$$
y^{2}=x_{m}^{4}+\frac{x_{m} \sinh x_{m} \tilde{d}\left[1-G\left(x_{m}, z\right)\right]}{\cosh x_{m} \tilde{d}-\cos z \tilde{d}}-\frac{1}{4 \tilde{\tau}^{2}} .
$$

We have denoted $y=\omega / E_{s}$, and $z=q_{z} / q_{s}$ in the above expressions. The total plasmon density of states for a superlattice CBG is shown in Fig. 4. In the RPA (Fig. 4A) an increasing disorder reduces the density of states $\rho(\omega)$ and smoothes the peak structure seen for the $1 / \tau=0$ case. The correlation effects included in the form of local-field correction, $G\left(q, q_{z}\right)$, pronounces that peak, but as the disorder is increased results similar to the RPA case are obtained. The density of states behaves linearly for small $\omega$, which is also the case for electron superlattices [19].

Finally, we calculate the ground-state energy of the CBG superlattice. Employing the standard [17] manipulations we obtain, in Rydberg units (Ry),

$$
E=-\frac{\tilde{d}}{\pi r_{s}^{2}} \int_{0}^{r_{s}} d r_{s}^{\prime} r_{s}^{1 / 3} \gamma\left(d, r_{s}^{\prime}\right)
$$

where

$$
\gamma\left(d, r_{s}\right)=-\frac{1}{q_{s}^{2}} \int_{-\pi / d}^{\pi / d} d q_{z} \int_{0}^{\infty} d q \frac{\sinh q d}{\cosh q d-\cos q_{z} d}\left[S\left(q, q_{z} ; \tau\right)-1\right] .
$$

The function $\gamma\left(d, r_{s}\right)$ embodies correlation and disorder effects through the static structure factor $S\left(q, q_{z} ; \tau\right)$. In the RPA, where we set $G_{H}\left(q, q_{z}\right)=0, \gamma\left(d, r_{s}\right)$ is independent of $r_{s}$ and we obtain $E=-(3 / 4 \pi)$ $\tilde{d} r_{s}^{-2 / 3} \gamma(d)$. In Fig. 5A we show $-(4 / 3) E r_{s}^{2 / 3}$ as a function of $\tilde{d}$ for $\left(2 \tau E_{s}\right)^{-2}=1$ (sold), 0.1 (dashed), and 0 (dotted), respectively. As the degree of disorder is increased ( $\tau$ becomes small) the magnitude of the groundstate energy increases. For large superlattice period $\tilde{d}$ our results approach the strictly two-dimensional CBG limit. We next include the local-field effects in the static structure factor, hence in the ground-state energy. Figure 5B shows the ground-state energy as a function of $r_{s}$, with (solid) and without (dotted) disorder 

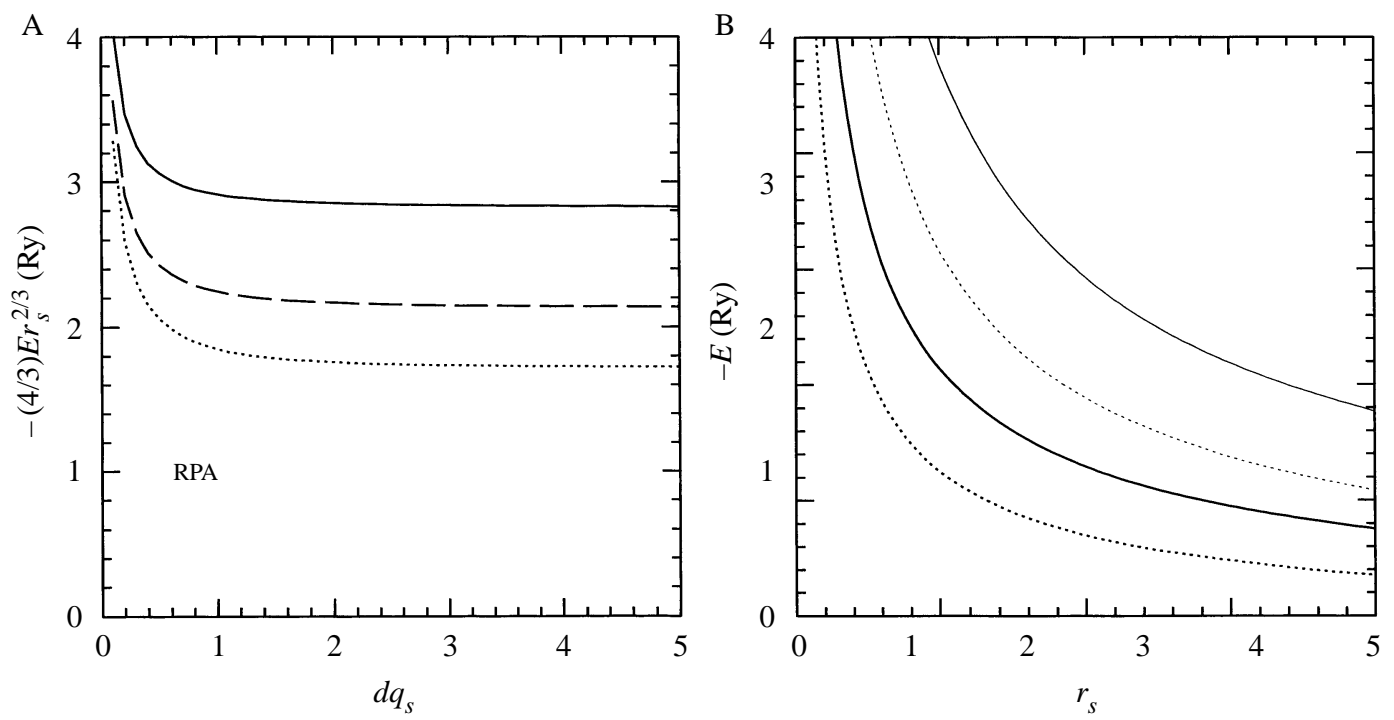

Fig. 5. A, The ground-state energy within the RPA as a function of the superlattice period for $\left(2 \tau E_{S}\right)^{-2}=1$ (solid), 0.1 (dashed), and 0 (dotted), respectively. B, The ground-state energy including the local-field corrections as a function of $r_{s}$. Solid and dotted curves are calculated with and without disorder $\left[\left(2 \tau E_{S}\right)^{-2}=1\right]$, respectively. Thick and thin curves are for $\tilde{d}=1$ and $\tilde{d}=0.5$, respectively.

effects. For the disordered case we set $\left(2 \tau E_{s}\right)^{-2}=1$. The thick and thin curves indicate $\tilde{d}=1$ and $\tilde{d}=$ 0.5 , respectively. We remark that the local-field correction $G\left(q, q_{z}\right)$ in the Hubbard approximation does not contain any disorder effects, and is valid only for small $r_{s}$. In view of this we expect the trends observed in Fig. 5B to be qualitatively correct. It would be desirable to study the disorder effects in the evaluation of the local-field correction, perhaps through the self-consistent scheme of Singwi et al. [21].

In conclusion, we have studied the collective excitations in a charged Bose gas superlattice in the presence of disorder whose effect has been modeled in the relaxation-time approximation. We have found that there is a critical wavevector below which plasmons do not propagate, as in the disordered electron gas case. An analytical expression is given for the static structure factor $S\left(q, q_{z} ; \tau\right)$ in the presence of disorder which may be useful for subsequent applications. The energy-loss functions $S\left(q, q_{z}, \omega ; \tau\right)$ is studied as a function of $\omega$ for fixed values of $q$ and $q_{z}$. Plasmon density of states is calculated for its relevance to charged Bose gas models of high- $T_{C}$ superconductors. The ground-state energy of the system is evaluated as a function of the superlattice period including the local-field and disorder effects.

Acknowledgements-This work was supported in part by the Scientific and Technical Research Council of Turkey (TUBITAK) under Grant No. TBAG-1662.

\section{References}

[1] A. S. Alexandrov and N. F. Mott, Phys. Rev. Lett. 71, 1075 (1993); Rep. Prog. Phys. 57, 1197 (1994); N. F. Mott, Adv. Phys. 39, 55 (1990).

[2] A. Gold, Physica C190, 483 (1992); Z. Phys. B81, 155 (1990).

[3] R. Mincas, J. Ranninger, and S. Robaszkiewicz, Rev. Mod. Phys. 62, 113 (1990).

[4] R. Friedberg, T. D. Lee, and H. C. Ren, Ann. Phys., NY 208, 149 (1991).

[5] T. Ando, A. B. Fowler, and F. Stern, Rev. Mod. Phys. 54, 437 (1982); C. Weisbuch and B. Vinter, Quantum Semiconductor Structures (Academic, New York, 1991). 
[6] L. D. Shvartsman and J. E. Golub, Bose-Einstein Condensation, edited by A. Griffin, D. W. Snoke, and S. Stringari (Cambridge, New York, 1995) p. 532.

[7] D. F. Hines and N. E. Frankel, Phys. Rev. B20, 972 (1979); M. L. Chiofalo, S. Conti, and M. P. Tosi, J. Phys.: Condens. Matter 8, 1921 (1996); S. Conti, M. L. Chiofalo, and M. P. Tosi, ibid. 6, 8795 (1994).

[8] B. Tanatar and A. K. Das, J. Phys.: Condens. Matter 7, 6065 (1995); ibid. 8, 1937 (1996).

[9] V. Apaja, J. Halinen, V. Haalonen, E. Krotscheck, and M. Saarela, Phys. Rev. B55, 12925 (1997); K. Tankeshwar, B. Tanatar, and M. P. Tosi, Phys. Rev. B57, 8854 (1998).

[10] R. K. Moudgil, P. K. Ahluwalia, and K. N. Pathak, Phys. Rev. B56, 14776 (1997); R. K. Moudgil, K. Tankeshwar, and K. N. Pathak, J. Phys.: Condens. Matter 11, 4645 (1999).

[11] G. F. Giuliani and J. J. Quinn, Phys. Rev. B29, 2321 (1984); D. J. W. Geldart, A. K. Das, and G. Gumbs, Solid State Commun. 60, 987 (1986); J. G. Cordes and A. K. Das, Solid State Commun. 80, 145 (1991).

[12] A. Gold, Phys. Rev. A33, 652 (1986); Z. Phys. B91, 501 (1993); K. Huang and H.-F. Meng, Phys. Rev. Lett. 69, 644 (1992).

[13] A. Gold, Z. Phys. B52, 1 (1983); ibid. 83, 429 (1991).

[14] P. B. Visscher and L. M. Falicov, Phys. Rev. B3, 2541 (1971); A. L. Fetter, Ann. Phys., NY 88, 1 (1974).

[15] A. Griffin and A. J. Pindor, Phys. Rev. B39, 11503 (1989); C. Zhang and N. Tzoar, Phys. Rev. A38, 5786 (1988); H. M. Miesenböck and M. P. Tosi, Z. Phys. B78, 255 (1990); W. B. da Costa, U. de Freitas, and N. Studart, Superlatt. Microstruct. 16, 67 (1994).

[16] A. Gold, Z. Phys. B86, 193 (1992); Phys. Rev. B47, 6762 (1993); Z. Phys. B101, 121 (1996).

[17] G. D. Mahan, Many Particle Physics (Plenum, New York, 1981); A. L. Fetter and J. D. Welecka, Quantum Theory of Many-Particle Systems (McGraw-Hill, New York, 1971); P. M. Platzman and P. A. Wolff, Waves and Interactions in Solid State Plasmas, Solid State Physics, Supplement 13 (Academic Press, New York, 1973).

[18] N. D. Mermin, Phys. Rev. B1, 2362 (1970); A. K. Das, J. Phys. F5, 2035 (1975).

[19] H. Morawitz, I. Bozovic, V. Z. Kresin, G. Rietveld, and D. van der Marel, Z. Phys. B90, 277 (1993).

[20] A. Gold, Z. Phys. B89, 1 (1992).

[21] K. S. Singwi, M. P. Tosi, R. H. Land, and A. Sjölander, Phys. Rev. 179, 589 (1968). 